\title{
Um estudo comparativo da freqüência de verbalização empática entre psicoterapeutas de diferentes abordagens teóricas
}

\section{A comparative study of emphatic verbalization frequency among therapists from different theoretical approaches}

\author{
Eliane Mary de Oliveira FALCONE \\ Débora Barbosa GIL² \\ Maria Cristina FERREIRA ${ }^{3}$
}

\begin{abstract}
Resumo
O estudo comparou a freqüência de verbalização empática entre dezesseis terapeutas distribuídos em quatro grupos de diferentes orientações: terapia centrada na pessoa, Gestalt-terapia, terapia cognitivo-comportamental e lacaniana. Cada terapeuta entrevistou a mesma cliente que apresentou a mesma queixa e o conteúdo das entrevistas foi avaliado por três juízes independentes e pela cliente. De acordo com a avaliação dos juízes, o grupo de terapia centrada na pessoa revelou freqüência de verbalização empática significativamente superior a dos grupos de Gestalt-terapia e terapia lacaniana e não significativamente superior a do grupo de terapia cognitivo-comportamental. Entretanto, com base na avaliação da cliente, a freqüência de verbalização empática do grupo de Gestalt-terapia foi significativamente superior à dos terapeutas das outras três abordagens, seguida do grupo de terapia cognitivo-comportamental, que também se revelou significativamente superior à do grupo de terapia centrada na pessoa e do de terapia lacaniana. O estudo confirma as divergências apontadas na literatura entre a empatia percebida pelo terapeuta e pelo cliente. São comentadas no artigo algumas limitações dessa pesquisa.
\end{abstract}

Unitermos: empatia; empatia terapêutica; relação terapêutica.

\begin{abstract}
The present study compared the frequency of empathic verbalization among 16 therapists allocated into four groups according to their theoretical orientation: Centered in the person, Gestalt therapy, Cognitive-Behavior Therapy, and Lacanian Therapy. Each therapist interviewed the same client who presented the same complaint. Interview contents were evaluated by three independent judges and by the client herself. According to judges' evaluations, centered in the person showed a significantly higher frequency of empathic verbalization compared to gestalt therapy and Lacanian Therapy, but not significantly higher than Cognitive-Behavior Therapy. However, based on the client's evaluations, the frequency of empathic verbalization of gestalt therapy was significantly higher than those three other approaches. In addition, Cognitive-Behavior Therapy frequency was higher than centered in the person and Lacanian Therapy. Results confirm the there is a weak relationship between empathy perceived by the therapist and client's perception mentioned in the literature. Some limitations of this study are pointed out.
\end{abstract}

Uniterms: empathy; therapeutic empathy; therapeutic relationship.

$\boldsymbol{\nabla r v \boldsymbol { V }}$

1 Universidade do Estado do Rio de Janeiro, Instituto de Psicologia. R. São Francisco Xavier, 524, $10^{\circ}$ andar, Maracanã, 20550-013, Rio de Janeiro, RJ, Brasil. Correspondência para/Correspondence to: E.M.O. FALCONE. E-mail: <falcone@uninet.com.br>.

2 Exército Brasileiro, Hospital Central do Exército, Seção de Psicologia. Niterói, RJ, Brasil.

3 Universidade Salgado de Oliveira, Programa de Pós-Graduação em Psicologia, Curso de Mestrado. Niterói, RJ, Brasil.

As autoras agradecem aos terapeutas participantes dessa pesquisa pela disponibilidade e boa vontade, cedendo o seu tempo de atendimento, com o único propósito de colaborar com o estudo. 
A relação entre a empatia manifestada pelo terapeuta e a eficácia do tratamento tem sido largamente apontada por vários estudos (Andersen, 2005; Burns \& Auerbach, 1996; Carkhuff, 1969; Goldstein \& Myers, 1991; Myers, 2003; Yip, 2005). Já a ausência de empatia e a postura defensiva do terapeuta prejudicam a aliança, impedindo o progresso do tratamento e exercendo um impacto nocivo sobre os sentimentos e a auto-estima dos pacientes (Henry, Schat \& Strupp, 1990, citado por Burns \& Auerbach, 1996). Além disso, pesquisas que investigaram os aspectos comuns entre terapeutas de diferentes abordagens teóricas encontraram que esses profissionais compartilham da mesma opinião no que diz respeito aos efeitos terapêuticos positivos da empatia (Goldstein \& Myers, 1991; Reisman, 1986; Safran, 2002).

O reconhecimento dos efeitos terapêuticos da empatia tem motivado a criação de definições mais detalhadas desse construto, bem como de programas de treinamento que visam desenvolver essa habilidade no terapeuta (Carkhuff, 1969; Egan, 1994).

No campo da psicoterapia, a obra mais relevante sobre a empatia foi a de Carl Rogers, para quem o termo implica "perceber o marco de referência interior da outra pessoa com precisão e com os componentes emocionais que the pertencem, como se fosse essa pessoa, porém sem perder nunca a condição de 'como se' " (Rogers, 1959, p.210).

Estudos recentes sugerem que a empatia engloba componentes cognitivos, afetivos e comportamentais. O componente cognitivo caracteriza-se por uma capacidade de compreender, de forma acurada, a perspectiva e os sentimentos dos outros (Ickes, 1997; Karniol, 1982; Long \& Andrews, 1990; Zillman, 1991), permitindo àquele que percebe, estender o seu entendimento além do significado aparente imediato das palavras e ações de outras pessoas (Ickes, Marangoni \& Garcia, 1997).

O componente afetivo da empatia caracteriza-se por sentimentos de compaixão e simpatia pela outra pessoa, além de preocupação com o seu bem-estar, não implicando necessariamente experimentar os mesmos sentimentos do outro. Em vez de "sentir" o que o cliente está sentindo, o terapeuta experimenta um entendimento do que é sentido pelo cliente. Assim, a 452 empatia não é somente um entendimento intelectual, mas também um sentimento ou um processo experiencial de entendimento (Greenberg \& Elliott, 1997).

O componente comportamental da empatia consiste em transmitir um reconhecimento explícito e uma elaboração dos sentimentos e da perspectiva da outra pessoa, de tal maneira que ela se sinta compreendida e que isso lhe ajude a obter um maior entendimento acerca de seu estado interno (Barrett-Lennard, 1997; Egan, 1994; Falcone, 1999, 2003).

Durante uma interação, a habilidade empática do terapeuta ocorre em duas etapas. Na primeira, ele está envolvido em compreender os sentimentos e perspectivas do pacientee, de algum modo, experienciar o que está acontecendo com ele naquele momento. A segunda etapa consiste em comunicar esse entendimento de forma sensível (Barrett-Lennard, 1997; Greenberg \& Elliott, 1997). A compreensão empática inclui prestar atenção e ouvir sensivelmente. A comunicação empática inclui expressar entendimento, verbal ou não verbal, de forma sensível.

A função da verbalização empática é fazer com que a outra pessoa se sinta compreendida, além de ajudar a explorar suas preocupações de modo mais completo. Embora o terapeuta possa sinalizar, de forma não verbal, compreensão, aceitação e acolhimento, ao prestar atenção e ouvir sensivelmente (por exemplo, acenando com a cabeça, usando vocalizações breves tais como "sim", "hum-hum"), é através da verbalização empática que se pode avaliar a acuidade do entendimento do terapeuta (Falcone, 1999, 2003; Ickes, Marangoni \& Garcia, 1997).

Burleson (1985) propõe que as verbalizações mais sensíveis ou empáticas são aquelas que se focalizam nos sentimentos e pensamentos da pessoa-alvo, sem julgar, descrevendo, explicando e validando esses sentimentos e pensamentos. As verbalizações menos sensíveis ou menos empáticas tendem a focalizar o evento em si, impõem o próprio ponto de vista, desconsiderando ou ignorando os sentimentos e a perspectiva da outra pessoa.

O terapeuta pode verbalizar entendimento empático de várias maneiras. Greenberg e Elliott (1997) identificaram seis formas de responder em psicoterapia: entendimento, evocação, exploração, complementação comunicativa, conjectura e interpretação. Dessas, as 
quatro primeiras são consideradas mais empáticas, uma vez que focalizam os sentimentos e perspectivas do cliente. Já a conjectura e a interpretação apresentam níveis mais elevados de inferência e se baseiam em construtos teóricos. Partindo da classificação de Greenberg e Elliott (1997) e de outros estudos sobre verbalização empática (Bohart \& Tallman, 1997; Burleson, 1985; Linehan, 1997), Falcone (2003) propõe cinco formas de verbalização empática e cinco formas de verbalização não empática do terapeuta, as quais serão especificadas em detalhes mais adiante.

A função da empatia é vista de forma diferente por diferentes abordagens. Na terapia centrada na pessoa, por exemplo, a empatia envolve a focalização nos significados e experiências do cliente, momento a momento, acompanhada de freqüentes respostas de entendimento. Assim, o terapeuta tenta entender, sem julgar, o ponto de referência do cliente a cada momento, comunicando esse entendimento de volta, em uma sintonia comunicativa (Bohart \& Greenberg, 1997). Ele é apenas um facilitador de exploração e um companheiro na busca dessa exploração. Em vez de conduzir o cliente, o encoraja a ir onde ele teme ir. A descoberta do cliente é o que leva à sua reorganização interna (Greenberg \& Elliot, 1997).

De acordo com o pensamento psicanalítico, o entendimento empático facilita o processo terapêutico ao tornar os pacientes mais abertos para receber interpretações, além de facilitar o insight (Maclsaac, 1997). Em vez de utilizar a sintonia comunicativa característica da abordagem centrada na pessoa, o terapeuta psicodinâmico procura entrar na experiência do cliente para compreender como ele é, como experimenta e se comporta no mundo em um sentido geral. Para isso, o analista pode adquirir informação além do que o cliente está comunicando no momento (Bohart \& Greenberg, 1997).

O enfoque cognitivo-comportamental tem ressaltado a importância da empatia do terapeuta como um recurso fundamental para facilitar a adesão ao tratamento (Beck, Rush, Shaw \& Emery,1982). Leahy (2001) sugere que algumas técnicas cognitivas possam ser invalidantes para o cliente e geram resistência. Nesse momento, o terapeuta deve abrir mão da focalização na mudança, sendo empático com os sentimentos e pensamentos do cliente (Leahy, 2001; Linehan, 1997).
Entretanto o foco exclusivo na empatia como agente de mudança é questionado pelos terapeutas cognitivos, uma vez que fortalece o papel de vítima exercido pelo cliente, contribuindo para sua tendência em manter o status quo (Leahy, 2001; Linehan, 1997; Newman, 2002).

Pearls (1977) propõe três caminhos para o Gestalt-terapeuta na interação com o cliente: 1) simpatia ou envolvimento no campo total; 2) empatia, em que o interesse do terapeuta se centra exclusivamente em torno do paciente e suas reações e 3) apatia ou desinteresse. $\mathrm{O}$ autor afirma que há bons motivos para se preferir a empatia à simpatia, visto que essa última pode fazer com que o terapeuta se incline a dar todo o apoio ambiental que o cliente demanda ou, caso não o faça, tornar-se culpado ou mesmo defensivo. Todavia deve-se dar preferência a atitudes que combinem simpatia e frustração (dos desejos de suporte ambiental do cliente), já que pode não acontecer um contato verdadeiro na empatia. Nesse sentido, a empatia deve ser valorizada, porém não constitui o aspecto mais importante ou mesmo eficaz no processo terapêutico (Perls, 1977). A Gestalt-terapia também tem sido incluída no grupo de terapias que enfatizam o trabalho terapêutico nos níveis emocional e experiencial (Bohart \& Greenberg, 1997).

A avaliação da empatia através da verbalização do terapeuta na interação com o cliente tem sido um recurso sugerido, uma vez que através do conteúdo verbal se pode inferir a escuta e o entendimento do clínico (Greenberg \& Elliott, 1997; Ickes, Marangoni \& Garcia,1997). Entretanto os critérios de avaliação não devem se basear apenas no que os terapeutas consideram sobre o que é ou não empático. Bachelor (1988) e Bohart e Greenberg (1997) apontam estudos demonstrando que as correlações entre o nível de empatia percebido pelo cliente e pelo terapeuta são relativamente baixas. Outras pesquisas têm sugerido que a empatia percebida por terapeutas, professores, supervisores ou colegas geralmente não tem sido relacionada com aquela que é experimentada pelo paciente e que os pacientes podem ser melhores avaliadores da qualidade da empatia terapêutica do que os próprios terapeutas. Além disso, as avaliações dos pacientes têm maior probabilidade de prever a recuperação clínica (Burns \& Auerbach, 1996). Assim, a percepção do paciente deve ser considerada na avaliação da empatia do terapeuta. 
As contribuições extraídas da literatura sobre diferentes tipos de verbalização empática e de diferentes formas de ver a empatia sugerem que o tipo de abordagem pode influenciar na freqüência e no tipo de verbalização empática dos terapeutas. Considerando-se, por exemplo, que na sintonia empática o terapeuta está focalizado na expressão de entendimento momento a momento, sendo esse o principal recurso terapêutico, pode-se especular que os terapeutas de abordagem centrada na pessoa devem expressar verbalizações empáticas na interação com os seus clientes em uma freqüência superior aos terapeutas de outros enfoques em que a empatia é vista como um recurso facilitador do processo terapêutico. Partindo dessas considerações, o objetivo desse estudo foi avaliar se o tipo de abordagem adotado pelo terapeuta interfere no conteúdo e na freqüência de suas verbalizações empáticas.

\section{Método}

Participaram como sujeitos dessa pesquisa 16 psicólogos clínicos (12 do sexo feminino e quatro do sexo masculino), distribuídos em quatro grupos de quatro, de acordo com suas orientações teóricas. Os participantes eram residentes na cidade do Rio de Janeiro, na faixa etária entre os 35 e 64 anos. Todos esses profissionais possuíam no mínimo dez anos de experiência como psicoterapeutas e atendiam baseados em orientações lacaniana (TL), centrada na pessoa (TCP), gestáltica (GT) e cognitivo-comportamental (TCC).

O material utilizado consistiu de: 1) um gravador portátil Panasonic, modelo RQ-L 309, utilizado para gravar as sessões terapêuticas; 2) consentimento livre e esclarecido contendo explicações sobre a pesquisa, além de compromisso com o sigilo sobre as fitas gravadas, bem como sobre a identidade dos terapeutas.

O primeiro contato com os terapeutas se deu por telefone, quando foram convidados a participar da pesquisa e informados de que deveriam conduzir uma única sessão terapêutica simulada, com a duração de 40 minutos. Foi também esclarecido aos participantes que a sessão seria gravada em fita cassete. Uma estudante de psicologia, que se propôs a colaborar com a pesquisa, atuaria como "cliente", revelando em todas as entrevistas o mesmo problema pessoal e verdadeiro. A partir do relato da cliente colaboradora, os terapeutas deveriam conduzir a sessão de acordo com as suas orientações teóricas.

Após os terapeutas assinarem o consentimento livre e esclarecido, cujo conteúdo atendia às exigências éticas de uma pesquisa, a sessão iniciava com a cliente relatando o seu problema: experimentar ansiedade e insegurança elevadas diante de situações envolvendo desempenhar em público (por ex., apresentar-se em seminários, dar aulas etc.). Depois de gravadas, as entrevistas foram transcritas e avaliadas por três juízes cegos para os tipos de abordagem e previamente treinados para avaliar e categorizar as verbalizações dos terapeutas. Após atingirem uma concordância satisfatória $(0,80)$ ao avaliarem trechos de sessões psicoterápicas extraídas da literatura, os juízes iniciaram a avaliação das entrevistas transcritas.

A verbalização de cada terapeuta foi avaliada pelos juízes de acordo com os critérios propostos por Falcone (2003) sobre os tipos de verbalização dos terapeutas. No primeiro critério, chamado de dimensão, a verbalização era considerada empática (VE) ou não empática (VN). No segundo critério, chamado de categoria, as verbalizações foram avaliadas de acordo com os subtipos de VE e de VN.

A dimensão VE incluiu as seguintes categorias: 1) Inespecífica, na qual a empatia do terapeuta se expressa em sentido global, não se enquadrando em nenhuma das categorias; 2) Entendimento empático ou reflexão acurada, em que o terapeuta busca um entendimento compartilhado, baseado na experiência explícita ou implícita do cliente, possibilitando e aceitando discordâncias do mesmo (ex:: "Você se sente oprimida por tantas pressões e obrigações e necessita se libertar disso para obter alívio. Mas, por outro lado, tentar obter alívio faz você sentir que não é responsável. Faz sentido?"); 3) Evocação empática, na qual o entendimento é expresso por metáforas, linguagem expressiva, imagem evocativa ou desempenhando o papel do cliente (Ex:: "Sim, buscando alguém que the diga: 'não se preocupe, Cláudia, está tudo bem"I'); 4) Exploração empática, identificada quando o terapeuta transmite entendimento selecionando daquilo que é mais pungente, não claro, idiossincrático ou implícito na fala do cliente, para explorar algo novo ou ver de uma nova maneira (Ex:: "Há algo sobre estar sozinha agora que é muito difícil..."); 5) Complementação comunicativa, expressa pela 
complementação da frase do cliente, quando ele se esforça para explicar algo (Ex.:."... Como se tivesse levado um soco no estômago").

As categorias da dimensão VN incluíram: 1) Exploratória, quando o terapeuta focaliza o evento, explorando os dados a respeito, sem considerar ou valorizar os sentimentos e perspectivas do cliente (Ex.: "Você se lembra de ter se sentido rejeitada assim com outros relacionamentos?"); 2) Com transmissão do próprio ponto de vista, quando o terapeuta expõe o seu (Ex::"As pessoas só percebem que estão incomodando quando a gente reclama"); 3) Com base em teorias que norteiam a abordagem do terapeuta (Ex.: "Você ficou com raiva de sua professora porque ela lembrou sua mãe"); 4) Sugestões ou conselhos, quando o terapeuta sugere o que o cliente poderia fazer para superar ou resolver determinado problema (Ex:."Você já pensou na possibilidade de conversar com o professor após a aula?")

A cliente colaboradora também avaliou as verbalizações do terapeuta de acordo com um critério que continha cinco afirmativas, graduadas desde a mais empática até a menos empática. As afirmativas foram apresentadas da seguinte forma: A ("A verbalização do terapeuta captou com acuidade, sem julgar, o que eu estava pensando e sentindo, de tal maneira que eu passei a ter uma compreensão mais clara do meu estado interno"); B ("A verbalização do terapeuta captou com acuidade, sem julgar, o que eu estava pensando e sentindo"); C ("A verbalização do terapeuta demonstrou aceitação/acolhimento/proximidade/afeto"); D ("A verbalização do terapeuta não se encaixou em nenhum dos critérios anteriores"); E ("A verbalização do terapeuta me deu a impressão de que ele não me compreendeu realmente"). As afirmativas A, B e C são consideradas empáticas e as afirmativas D e E são avaliadas como não empáticas.

\section{Resultados}

Os dados das avaliações foram tabulados e analisados estatisticamente. Foram calculados os coeficientes de concordância entre os três juízes para as classificações realizadas em função das categorias e das dimensões pelo cálculo dos coeficientes obtidos pelos três diferentes pares de avaliadores passíveis de serem formados e da média dos coeficientes assim obtidos. Os resultados indicaram um índice de concordância de 0,71 para as avaliações das verbalizações feitas de acordo com as categorias e de 0,88 para as avaliações feitas de acordo com as dimensões.

Em seguida, as verbalizações emitidas por cada terapeuta receberam uma única classificação por categoria e por dimensão, correspondentes às avaliações mais freqüentemente recebidas por parte dos três juízes, o que permitiu o cômputo da freqüência total de verbalizações obtidas em cada uma das entrevistas, em função das diversas categorias e dimensões de análise.

Posteriormente foram calculadas as freqüências e percentagens obtidas nas várias categorias de VE, segundo o tipo de abordagem do terapeuta (Tabela 1). A comparação dos resultados observados em cada uma dessas categorias pelas diferentes abordagens terapêuticas foi realizada através do teste de comparação entre proporções (Viera, 1994), tendo-se verificado a existência de diferenças significativas apenas entre as abordagens TCP e TCC ( $z=3,00 ; p<0,01)$ e as abordagens $\operatorname{TL} \operatorname{eTCC}(z=1,96 ; p<0,05)$, na categoria de entendimento empático, bem como entre as abordagens TCP e GT $(z=2,67 ; p<0,01)$, na categoria de complementação comunicativa. Nesse sentido, verificou-se que as verbalizações associadas ao entendimento empático foram significativamente mais freqüentes nas abordagens TCP e TL, quando comparadas à abordagem TCC, enquanto as verbalizações classificadas na categoria de complementação comunicativa foram emitidas em freqüência significativamente maior pelos terapeutas GT, quando comparados aos terapeutas TCP. Tais resultados devem, entretanto, ser vistos com cautela, na medida em que as classificações por categorias obtiveram um coeficiente de concordância relativamente baixo.

Procedimentos semelhantes foram empregados para a análise das freqüências e percentagens obtidas nas diferentes categorias de VN, segundo o tipo de abordagem do terapeuta (Tabela 2). Desse modo, a comparação entre as várias abordagens, consideradas duas a duas, evidenciou que as mesmas apresentaram diferenças quanto à freqüência de VN da categoria exploratória, com a abordagem TCC destacando-se como a de maior freqüência de verbalizações dessa 
natureza, tendo a mesma se diferenciado significativamente das modalidades TCP $(z=9,75 ; p<0,01) ; \mathrm{TL}$ $(z=3,75 ; p<0,01)$ e $G T(z=11,50 ; p<0,01)$. Já a abordagem $T L$, embora tenha apresentado freqüências de verbalizações um pouco menores que as observadas na abordagem TCC, ainda assim apresentou freqüências significativamente maiores que as abordagens TCP $(z=6,00 ; p<0,01)$ e $G T(z=7,75 ; p<0,01)$, que não se diferenciaram entre si quanto à emissão de verbalizações não empáticas de natureza exploratória.

No que diz respeito às VN com transmissão do ponto de vista do terapeuta, foi constatado que foram usadas com freqüência significativamente maior pelos terapeutas TL, em comparação aos TCC $(z=6,50 ; p<0,01)$ e $\mathrm{GT}(\mathrm{z}=3,60 ; p<0,01)$, que não se diferenciaram entre si no que tange ao uso dessa modalidade de verbalização. Em segundo lugar surgiu a TCP, que também apresentou um número de verbalizações significativamente maior que as classificadas na abordagem TCC $(z=5,00 ; p<0,01)$.
Por fim, foram observadas diferenças significativas entre as vocalizações não verbais, classificadas como VN por não preencherem os critérios de uma VE. As abordagens que apresentaram as maiores freqüências de emissão de verbalizações dessa natureza foram a GT e a TCP, que não se diferenciaram entre si, mas apresentaram freqüências significativamente maiores nesse quesito do que as abordagens $T L(z=10,50 ; p<0,01$ e $z=6,75 ; p<0,01$, respectivamente) eTCC ( $z=11,00 ; p<0,01$ e $z=9,750 ; p 0,01$, respectivamente).

Atendo-se às comparações entre as dimensões empática e não empática (Tabela 3), foi possível verificar que a abordagem TCP diferenciou-se significativamente das abordagens $\mathrm{TL}(\mathrm{z}=3,67 ; p<0,01)$ e $\mathrm{GT}(\mathrm{z}=4,00 ; p<0,01)$ por apresentar um maior número de VE do que as outras duas abordagens, que, em contrapartida, suscitaram maior freqüência de VN que a abordagem TCP. Já a abordagem TCC, embora tenha apresentado uma freqüência de VE inferior apenas à eliciada pela abordagem TCP, ainda assim não se diferenciou significativamente de nenhuma das outras abordagens terapêuticas.

Tabela 1. Categorias de verbalização empática segundo a abordagem do terapeuta, na avaliação dos juízes.

\begin{tabular}{|c|c|c|c|c|c|c|c|c|}
\hline \multirow{3}{*}{ Categorias de VE } & \multicolumn{8}{|c|}{ Tipo de abordagem do terapeuta } \\
\hline & \multicolumn{2}{|c|}{$\mathrm{TCP}$} & \multicolumn{2}{|c|}{ TL } & \multicolumn{2}{|c|}{ TCC } & \multicolumn{2}{|c|}{ GT } \\
\hline & $\mathrm{F}$ & $\%$ & $\mathrm{~F}$ & $\%$ & $F$ & $\%$ & $\mathrm{~F}$ & $\%$ \\
\hline Inespecífica & 0 & 0 & 1 & 6,2 & 1 & 3,1 & 0 & 0 \\
\hline Entendimento empático & 56 & 93,3 & 15 & 93,8 & 22 & 68,8 & 18 & 81,8 \\
\hline Evocação empática & 2 & 3,3 & 0 & 0 & 3 & 9,4 & 0 & 0 \\
\hline Exploração empática & 1 & 1,7 & 0 & 0 & 1 & 3,1 & 0 & 0 \\
\hline Complementação comunicativa & 1 & 1,7 & 0 & 0 & 5 & 15,6 & 4 & 18,2 \\
\hline Total & 60 & 100,0 & 16 & 100,0 & 32 & 100,0 & 22 & 100,0 \\
\hline
\end{tabular}

VE: verbalização empática; TL: teoria lacaniana; TCP: teoria centrada na pessoa; TCC: teoria cognitivo-comportamental; GT: Gestalt-teoria.

Tabela 2. Categorias de verbalização não-empática segundo a abordagem do terapeuta, na avaliação dos juízes.

\begin{tabular}{|c|c|c|c|c|c|c|c|c|}
\hline \multirow{3}{*}{ Categorias de VN } & \multicolumn{6}{|c|}{ Tipo de abordagem do terapeuta } & & \\
\hline & \multicolumn{2}{|c|}{$\mathrm{TCP}$} & \multicolumn{2}{|c|}{$\mathrm{TL}$} & \multicolumn{2}{|c|}{ TCC } & \multicolumn{2}{|c|}{ GT } \\
\hline & $\mathrm{F}$ & $\%$ & $\mathrm{~F}$ & $\%$ & $\mathrm{~F}$ & $\%$ & $\mathrm{~F}$ & $\%$ \\
\hline Exploratória & 128 & 46,6 & 161 & 71,2 & 231 & 86,2 & 147 & 40,2 \\
\hline Com transmissão do ponto de vista do terapeuta & 36 & 13,1 & 37 & 16,4 & 9 & 3,4 & 27 & 7,4 \\
\hline Com base em teorias ou experiência do terapeuta & 21 & 7,6 & 12 & 5,3 & 18 & 6,7 & 15 & 4,1 \\
\hline O terapeuta tenta dar sugestões ou conselhos & 0 & 0 & 3 & 1,3 & 0 & 0 & 2 & 0,5 \\
\hline $\begin{array}{l}\text { Vocalizações não-verbais que não preenchem } \\
\text { critérios de uma verbalização empática }\end{array}$ & 90 & 32,7 & 13 & 5,8 & 10 & 3,7 & 175 & 47,8 \\
\hline Total & 275 & 100,0 & 226 & 100,0 & 268 & 100,0 & 366 & 100,0 \\
\hline
\end{tabular}

456

VN: teoria-não empática; TL: teoria lacaniana; TCP: teoria centrada na pessoa; TCC: teoria cognitivo-comportamental; GT: Gestalt-teoria. 
Tabela 3. Verbalizações empáticas e não-empáticas segundo a abordagem do terapeuta, na avaliação dos juízes e da cliente.

\begin{tabular}{|c|c|c|c|c|c|c|c|c|}
\hline \multicolumn{9}{|l|}{ Avaliação dos juízes } \\
\hline \multirow{3}{*}{ Tipo de verbalização } & \multicolumn{8}{|c|}{ Tipo de abordagem do terapeuta } \\
\hline & \multicolumn{2}{|c|}{$\mathrm{TCP}$} & \multicolumn{2}{|c|}{$\mathrm{TL}$} & \multicolumn{2}{|c|}{ TCC } & \multicolumn{2}{|c|}{ GT } \\
\hline & $\mathrm{F}$ & $\%$ & $\mathrm{~F}$ & $\%$ & $\mathrm{~F}$ & $\%$ & $\mathrm{~F}$ & $\%$ \\
\hline VE & 60 & 17,9 & 16 & 6,6 & 32 & 10,7 & 22 & 5,7 \\
\hline VN & 275 & 82,1 & 226 & 93,4 & 268 & 89,3 & 366 & 94,3 \\
\hline Total & 335 & 100,0 & 242 & 100,0 & 300 & 100,0 & 388 & 100,0 \\
\hline \multicolumn{9}{|l|}{ Avaliação da cliente } \\
\hline \multirow{3}{*}{ Tipo de verbalização } & \multicolumn{8}{|c|}{ Tipo de abordagem do terapeuta } \\
\hline & \multicolumn{2}{|c|}{$\mathrm{TCP}$} & \multicolumn{2}{|c|}{ TL } & \multicolumn{2}{|c|}{ TCC } & \multicolumn{2}{|c|}{ GT } \\
\hline & $\mathrm{F}$ & $\%$ & $\mathrm{~F}$ & $\%$ & $\mathrm{~F}$ & $\%$ & $\mathrm{~F}$ & $\%$ \\
\hline$V E$ & 232 & 57,6 & 145 & 52,7 & 255 & 75,7 & 371 & 85,3 \\
\hline VN & 171 & 42,4 & 130 & 47,3 & 82 & 24,3 & 64 & 14,7 \\
\hline Total & 403 & 100,0 & 275 & 100,0 & 337 & 100,0 & 435 & 100,0 \\
\hline
\end{tabular}

VN: teoria-não empática; VE: verbalização empática; TL: teoria lacaniana; TCP: teoria centrada na pessoa; TCC: teoria cognitivo-comportamental; GT: Gestalt-teoria.

Tabela 4. Categorias de verbalização empática segundo a abordagem do terapeuta, na avaliação da cliente.

\begin{tabular}{|c|c|c|c|c|c|c|c|c|}
\hline \multirow{3}{*}{ Categorias de Verbalização } & \multicolumn{8}{|c|}{ Tipo de abordagem do terapeuta } \\
\hline & \multicolumn{2}{|c|}{$\mathrm{TCP}$} & \multicolumn{2}{|c|}{$\mathrm{TL}$} & \multicolumn{2}{|c|}{ TCC } & \multicolumn{2}{|c|}{ GT } \\
\hline & $\mathrm{F}$ & $\%$ & $\mathrm{~F}$ & $\%$ & $\mathrm{~F}$ & $\%$ & $\mathrm{~F}$ & $\%$ \\
\hline A & 17 & 7,3 & 21 & 14,5 & 12 & 4,7 & 8 & 2,2 \\
\hline B & 46 & 19,8 & 29 & 20,0 & 50 & 19,6 & 40 & 10,8 \\
\hline C & 169 & 72,9 & 95 & 65,5 & 193 & 75,7 & 323 & 87,0 \\
\hline Total & 232 & 100,0 & 145 & 100,0 & 255 & 100,0 & 371 & 100,0 \\
\hline
\end{tabular}

TL: teoria lacaniana; TCP: teoria centrada na pessoa; TCC: teoria cognitivo-comportamental; GT: Gestalt teoria.

As freqüências e percentagens das avaliações realizadas pela cliente foram também computadas, permitindo, assim, a comparação das diferentes abordagens terapêuticas também nesse aspecto, mediante a utilização dos mesmos procedimentos já citados. No que diz respeito às categorias de VE (Tabela 4), os dados obtidos evidenciaram que, na categoria A, associada à apreensão acurada do estado interno da cliente por parte do terapeuta, ocorreram diferenças significativas somente entre a abordagem $\mathrm{TL}$, por um lado, e, por outro, as abordagens TCC ( $z=3,00 ; p<0,01)$ e $G T(z=6,00$; $p<0,01)$. Tais diferenças sugeriram que as verbalizações classificadas na categoria A surgiram com freqüência significativamente maior entre os terapeutas $T L$ do que entre os TCC ou GT.
Na categoria B (verbalização acurada sem julgamento), as abordagens TL, TCC eTCP apresentaram freqüências equivalentes e todas elas foram significativamente superiores às obtidas pela abordagem GT ( $z=3,00 ; p<0,01$, em todos os três pares de comparações), que apresentou freqüências bem mais baixas nesse quesito. Já na categoria C, ocorreu o oposto, na medida em que as freqüências das verbalizações eliciadas pela abordagem GT revelaram-se significativa-mente superiores às obtidas nas modalidades TCP $(z=4,67$; $p<0,01)$, TL $(z=5,25 ; p<0,01)$ e TCC $(z=3,67 ; p<0,01)$. Contrariamente ao observado nas categorias de $V E$, porém, a análise das categorias de VN avaliadas pela cliente (Tabela 5) evidenciou que em nenhuma dessas categorias ocorreram diferenças significativas entre as diversas abordagens terapêuticas incluídas no estudo. 
Tabela 5. Categorias de verbalização não-empática segundo a abordagem do terapeuta, na avaliação da cliente.

\begin{tabular}{|c|c|c|c|c|c|c|c|c|}
\hline \multirow{3}{*}{ Categorias de Verbalização } & \multicolumn{8}{|c|}{ Tipo de abordagem do terapeuta } \\
\hline & \multicolumn{2}{|c|}{$\mathrm{TCP}$} & \multicolumn{2}{|c|}{$T L$} & \multicolumn{2}{|c|}{ TCC } & \multicolumn{2}{|c|}{ GT } \\
\hline & $\mathrm{F}$ & $\%$ & $\mathrm{~F}$ & $\%$ & $\mathrm{~F}$ & $\%$ & $\mathrm{~F}$ & $\%$ \\
\hline D & 142 & 83 & 105 & 80,8 & 76 & 92,7 & 56 & 87,5 \\
\hline$E$ & 29 & 17 & 25 & 19,2 & 6 & 7,3 & 8 & 12,5 \\
\hline Total & 171 & 100 & 130 & 100,0 & 82 & 100,0 & 64 & 100,0 \\
\hline
\end{tabular}

VN: teoria-não empática; VE: verbalização empática; TL: teoria lacaniana; TCP: teoria centrada na pessoa; TCC: teoria cognitivo-comportamental; GT: Gestalt-teoria.

No que tange à comparação das freqüências observadas nas dimensões empática e não empática, segundo a percepção da cliente (Tabela 3), várias diferenças surgiram. Assim a abordagem com maior incidência de verbalizações empáticas foi a GT, seguida pelaTCC, tendo as duas diferido significativamente entre si $(z=3,00 ; p<0,01)$, além de ambas terem se diferenciado significativamente tanto da abordagem $\mathrm{TL}(\mathrm{z}=10,67$; $p<0,01$ e $z=5,75 ; p<0,01$, respectivamente) quanto $d a$ abordagem TCP ( $z=9,00 ; p<0,01$ e $z=6,00 ; p<0,01$, respectivamente), com essas duas outras modalidades terapêuticas (TL e TCP) demonstrando, conseqüentemente, uma incidência menor de VE, além de não terem se diferenciado entre si. Em contrapartida, as abordagens TL e TCP tiveram uma incidência significativamente maior de VN em comparação às outras duas abordagens terapêuticas consideradas.

\section{Discussão}

A partir da avaliação dos juízes, os terapeutas TCP apresentaram maior freqüência de VE e menor freqüência de $V N$ do que os terapeutas das outras três abordagens, sendo essas diferenças, tanto para VE quanto para VN, estatisticamente significantes em relação aos terapeutas TL e GT. Tais resultados são concordantes com a hipótese levantada anteriormente, baseada na afirmação de que o terapeuta TCP expressa entendimento empático momento a momento na interação com o paciente, em uma sintonia comunicativa, focalizando principalmente a auto-aceitação desse último (Bohart \& Greenberg, 1997). Logo, sendo a empatia o elemento chave de exploração e de autodescoberta para a ocorrência da mudança, pode-se deduzir que a verbalização empática na intervenção terapêutica baseada na abordagem TCP tende a ser mais freqüente do que nas abordagens $T L$, GT eTCC, as quais consideram a empatia do terapeuta um elemento facilitador da intervenção psicoterápica e não o principal recurso de mudança, conforme o que foi apresentado anteriormente (Bohart \& Greenberg, 1997; Leahy, 2001; Linehan, 1997).

Com relação às categorias de $\mathrm{VE}$, verificou-se que o entendimento empático constituiu o tipo de verbalização mais freqüente entre os terapeutas de todas as abordagens, em comparação com as outras categorias, sugerindo haver uma preferência entre os terapeutas desta amostra por essa forma de verbalização. Cabe ressaltar que os terapeutas TL eTCP se destacaram significativamente dos outros dois grupos no que diz respeito a essa modalidade de VE.

A alta freqüência de VN do tipo exploratória também se mostrou predominante em relação às outras categorias de VN entre os terapeutas de todas as abordagens neste estudo. Uma possível razão para esse resultado se deve ao fato de que eles estavam realizando uma primeira entrevista, o que demanda uma tendência maior para a busca de dados sobre o cliente. A freqüência significativamente mais elevada de verbalização exploratória entre os terapeutas cognitivo-comportamentais pode ser explicada pela maior demanda dessa abordagem no sentido de explorar dados nas primeiras entrevistas com o objetivo de realizar uma formulação clínica antes de iniciar o processo terapêutico (Kirk, 1997).

Terapeutas $T L$ também apresentaram freqüência significativamente maior de VN do tipo exploratório do que os do grupo TCP e GT. Além disso, eles se destacaram na manifestação de verbalizações do tipo com transmissão do ponto de vista do terapeuta. 
Terapeutas TCP e GT apresentaram maior freqüência de vocalizações. Embora as vocalizações estivessem categorizadas no grupo das VN, por não refletirem claramente compreensão acurada do conteúdo do discurso do cliente, essas parecem funcionar como uma forma de validação não verbal da fala do paciente e, nesse sentido, podem ser facilitadoras do vínculo terapêutico. Em outras palavras, elas poderiam ser mais bem classificadas como expressão de um componente afetivo da empatia.

Os dados referentes às categorias de VE e de VN devem ser considerados com cautela, uma vez que a concordância entre os juízes nesse tipo de avaliação foi relativamente baixa.

A avaliação da empatia dos terapeutas a partir da percepção da cliente apresentou resultados bem diferentes daqueles avaliados pelos juízes. Assim, os terapeutas do grupo GT se revelaram com uma freqüência de VE significativamente superior às das outras três abordagens, seguidos dos terapeutas do grupo TCC. Esses resultados não são concordantes com a hipótese mencionada nesse estudo, que sugere haver maior freqüência de manifestação empática entre terapeutas TCP.

Outro dado importante extraído da avaliação da cliente refere-se às categorias de VE apresentadas pelos terapeutas deste estudo. Os terapeutas GT manifestaram freqüência significativamente superior aos das outras três escolas de verbalizações classificadas na categoria C, que reflete demonstração de aceitação, acolhimento, proximidade e afeto, e que se encaixa no componente afetivo da empatia.

Em contrapartida, os terapeutas do grupo TL foram os que apresentaram freqüência significativamente superior de verbalizações da categoria A, que reflete apreensão acurada do estado interno da cliente, que está além daquilo que é entendido por ela. Esse resultado é concordante com as afirmações de Bohart e Greenberg (1997) sobre a busca, por parte do terapeuta psicodinâmico, da informação que está além do que o cliente comunica no momento.

Na categoria B, que também se caracteriza pela percepção acurada do terapeuta, porém com um nível mais baixo de inferência, os terapeutas GT apresentaram freqüência de verbalização significativamente inferior a dos terapeutas das outras três abordagens. Assim, a partir da percepção da cliente, os terapeutas GT tenderam a manifestar a empatia dentro de um conteúdo mais afetivo, enquanto os terapeutas das outras abordagens manifestaram a empatia de forma predominantemente cognitiva. Tais resultados confirmam o que foi mencionado anteriormente sobre a presença do componente afetivo, experiencial, característico na Gestalt-terapia (Bohart \& Greenberg, 1997; Perls, 1973).

A ausência de relação entre as avaliações dos juízes e dos terapeutas dessa pesquisa é concordante com os estudos apontados por Bachelor (1988), Bohart e Greenberg (1997) e por Burns e Auerbach (1996). Cabe ressaltar, no entanto, que o grupo TCC se manteve no segundo lugar na freqüência de verbalizações da dimensão empática, tanto na avaliação dos juízes quanto na da cliente.

Embora a baixa relação entre as avaliações do cliente e do terapeuta quanto à empatia terapêutica seja reconhecida em vários estudos, algumas limitações dessa pesquisa podem ter contribuído para essa diferença. Primeiro, o número limitado da amostra pode ter interferido nos resultados. Segundo, a avaliação do cliente foi feita por apenas uma pessoa. Terceiro, de modo diferente dos juízes, a cliente teve acesso a informações não verbais dos terapeutas, o que pode ter interferido nas suas avaliações.

Sabe-se que as manifestações não verbais exercem uma influência muito grande na percepção do comportamento social e que algumas vezes os sinais não verbais superam a comunicação verbal (Argyle, 1987). Os juízes, por sua vez, avaliaram apenas o conteúdo verbal através das transcrições das verbalizações dos terapeutas, sem que tivessem qualquer contato com eles.

Deve-se ressaltar ainda que a avaliação da freqüência das verbalizações dos terapeutas nesse estudo não permite concluir que os participantes dessa amostra manifestaram pouca ou muita empatia na interação com a cliente. Além disso, a presença de verbalizações do tipo não empática dos terapeutas não indica necessariamente uma menor qualidade nas suas interações com a cliente. Embora a verbalização empática possa cumprir uma função central ou facilitadora da interação terapeuta-cliente, dependendo da abordagem (Barrett-Lennard, 1997; Greenberg \& Elliott, 1997), 
ela não constitui o único modo de atuação na relação de ajuda (Egan, 1994).

Alguns clientes se mostram sensíveis a uma postura excessivamente investigatória por parte do terapeuta, especialmente no início do tratamento. Nesse caso, a expressão empática de entendimento pode amenizar os efeitos negativos de uma entrevista que focaliza a obtenção de dados, facilitando a auto-revelação e a adesão ao tratamento.

Outra questão a ser ressaltada nessa pesquisa refere-se ao baixo nível de gravidade do problema apresentado pela cliente. Os terapeutas de qualquer abordagem teórica conseguem mais facilmente ser empáticos com clientes geralmente chamados de"normais" (que não se revelam tão resistentes, são mais cooperativos etc.), e apresentam mais dificuldades em lidar com clientes chamados de "difíceis", por apresentarem transtornos crônicos ou de personalidade (Beck \& Freeman, 1993). Esses últimos demandam mais paciência e empatia de seus terapeutas, uma vez que se comportam freqüentemente nas sessões de maneira hostil, interpretando mal, ironizando ou depreciando o terapeuta ou o seu trabalho (Burns \& Auerbach, 1996; Falcone, 2004; Safran, 2002). Tais comportamentos manifestados por esses clientes são estressantes para o terapeuta e não raro despertam nele reações hostis, que se tornam prejudiciais para o cliente (Safran, 2002). Assim, a empatia do terapeuta tem sido considerada como fundamental, principalmente no tratamento de pacientes com transtorno borderline de personalidade (Burns \& Auerbach, 1996; Safran, 2002; Young, Klosko \& Weishaar, 2003). É possível que uma pesquisa que compare verbalizações de terapeutas no atendimento a pacientes difíceis possa trazer resultados mais conclusivos do que o que revelou o presente estudo, uma vez que iria demandar maior esforço por parte dos participantes em verbalizar de forma empática.

Este estudo revelou que os terapeutas TCP manifestaram maior freqüência de verbalizações empáticas, sendo essa significativamente superior à dos terapeutas TL e GT, a partir da avaliação dos juízes, baseada em critérios extraídos da literatura para julgar comportamento empático versus não empático. Já a partir da avaliação da cliente, que julgou a empatia dos terapeutas baseada em sua percepção de ser ou não compreendida, os terapeutas GT apresentaram freqüência significativamente superior às das outras três orientações teóricas, assim como os terapeutas TCC, que também mostraram freqüência significativamente superior aos TCP eTL.

As avaliações dos juízes e da cliente, embora diferentes, sugerem que o tipo de abordagem influencia na freqüência, assim como em algumas categorias de VE ou de VN do terapeuta. Entretanto deve-se ter cuidado na generalização desses resultados. Considerando-se o tamanho restrito dessa amostra, não se pode assegurar que as orientações teóricas dos terapeutas, e não as suas características pessoais, tenham influenciado as verbalizações avaliadas nesse estudo.

Outro aspecto relevante baseado nos resultados desse trabalho refere-se à possibilidade da empatia ser manifestada pelo terapeuta com uma predominância do componente cognitivo (através de percepção acurada do estado interno do cliente) ou do componente afetivo (através de demonstração de calor, acolhimento, aceitação e afeto). Alguns clientes podem se sentir mais ou menos compreendidos por seus terapeutas, dependendo de suas demandas. Assim, os clientes que buscam um entendimento de seus problemas mais orientado para o insight poderão se sentir mais compreendidos pelos terapeutas que manifestam empatia acurada (cognitiva). Já os clientes que necessitam de validação ou de "ventilação" dos seus sentimentos podem requerer mais a empatia afetiva do terapeuta. Mais estudos são necessários para a verificação dessas especulações.

Algumas limitações dessa pesquisa devem ser consideradas. Sugere-se que futuros estudos apresentem:a) maior número de terapeutas; b) avaliação da comunicação não verbal dos terapeutas; c) maior número de clientes e que sejam portadores de problemas mais graves.

\section{Referências}

Andersen, D. T. (2005). Empathy, psychotherapy, integration, and meditation: a buddhist contribution to the common factors movement. Journal of Humanistic Psychology, 45 (4), 483-502.

Argyle, M. (1987). Bodily communication. London: Methuen.

Bachelor, A. (1988). How clients perceive therapist empathy: a content analysis of "received" empathy. Psychotherapy, 25 (2), 227-240.

Barrett-Lennard, G. T. (1997). The recovery of empathy: toward others and self. In A. C. Bohart \& L. S. Greenberg 
(Orgs.), Empathy reconsidered: new directions in psychotherapy (pp.103-121). Washington, DC: American Psychological Association.

Beck, A. T., Rush, A. J., Shaw, B. F., \& Emery, G. (1982). Terapia cognitiva da depressão. Rio de Janeiro: Zahar.

Beck, A., \& Freeman, A. (1993). Terapia cognitiva dos transtornos de personalidade. Porto Alegre: Artes Médicas.

Bohart, A. C., \& Greenberg, L. S. (1997). Empathy and psychotherapy: an introductory overview. In A. C. Bohart \& L. S. Greenberg (Orgs.), Empathy reconsidered: new directions in psychotherapy (pp.3-31). Washington, DC: American Psychological Association.

Bohart, A. C. \& Tallman, K. (1997). Empathy and the active client: an integrative, cognitive experiencial approach. In A. C. Bohart \& L. S. Greenberg (Orgs.), Empathy reconsidered: new directions in psychotherapy (pp.393-415). Washington, DC: American Psychological Association.

Burleson, B. R. (1985). The production of comforting messages: social cognitive foundations. Journal of Language and Social Psychology, 4 (3), 253-273.

Burns, D., \& Auerbach, A. (1996). Therapeutic empathy in cognitive-behavioral therapy: does it really make a difference? In P. M. Salkovskis \& S. Rachman (Orgs.), Frontiers of cognitive therapy (pp.135-163). New York: Guilford.

Carkhuff, R. R. (1969). Helping and human relations. New York: Holt, Rinehart \& Winston.

Egan, G. (1994). The skilled helper: a problem management approach to helping (5th ed.). Pacic Grove: Brooks/Cole.

Falcone, E. (1999). A avaliação de um programa de treinamento da empatia com universitários. Revista Brasileira de Terapia Comportamental e Cognitiva, 1 (1), 23-32.

Falcone, E. (2003). Empatia. In C. N. Abreu \& M. Roso (Orgs.), Psicoterapias cognitiva e construtivista. Novas fronteiras da prática clínica (pp.275-287). Porto Alegre: Artmed.

Falcone, E. (2004). A relação terapêutica. In P. Knapp (Org.), Terapia cognitivo-comportamental na prática psiquiátrica (pp.483-495). Porto Alegre: Artmed.

Goldstein, A. P., \& Myers, C. R. (1991). Relationship-enhancement methods. In F. H. Kanfer \& A. P. Goldstein (Orgs.), Helping people change: a textbook of methods (pp.19-65). New York: Pergamon Press.

Greenberg, L. S., \& Elliott, R. (1997). Varieties of empathic responding. In A. C. Bohart \& L. S. Greenberg (Org.), Empathy reconsidered: new directions in psychotherapy (pp.167-186). Washington, DC: American Psychological Association.

Ickes, W. (1997). Introduction. In W. Ickes (Org.), Empathic accuracy (pp.1-16). New York: The Guilford Press.

Ickes, W., Marangoni, C., \& García, S. (1997). Studying empathic accuracy in a clinically relevant context. In W. Ickes (Org.), Empathic accuracy (pp.282-310). New York: The Guilford Press.

Karniol, R. (1982). Settins, scripts, and self-schemata: a cognitive analysis of the development of prosocial behavior. In N. Eisenberg (Org.), The development of prosocial behavior (pp.251-278). New York: Academic Press.

Kirk, J. (1997). Avaliação cognitivo-comportamental. In K. Hawton, P. M. Salkovskis, J. Kirk \& D. M. Clark (Orgs.), Terapia cognitivo-comportamental para problemas psiquiátricos. São Paulo: Martins Fontes.

Leahy, R. L. (2001). Overcoming resistance in cognitive therapy. New York: Guilford.

Linehan, M. M. (1997). Validation and psychotherapy. In A. C. Bohart \& L. S. Greenberg (Orgs.), Empathy reconsidered: new directions in psychotherapy (pp.353-392). Washington, DC: American Psychological Association.

Long, E. C. J., \& Andrews, D. W. (1990). Perspective taking as a predictor of marital adjustment. Journal of Personality and Social Psychology, 59 (1), 126-131.

Maclsaac, D. S. (1997). Empathy: Heinz Kohut's contribution. In A. C. Bohart \& L. S. Greenberg (Orgs.), Empathy reconsidered: new directions in psychotherapy (pp. 245-264). Washington, DC: American Psychological Association.

Myers, S. (2003). Relational healing: to be understood and to understand. Journal of Humanistic Psychology, 43 (1), 86-104.

Newman, C. F. (2002). A cognitive perspective on resistance in psychotherapy.JCLP/In Session: Psychotherapy in Practice, 58 (2), 165-174

Pearls, F. (1977). A abordagem gestáltica é testemunha ocular da terapia. Rio de Janeiro: Zahar.

Reisman, J. M. (1986). Psychotherapy as a professional relationship. Professional Psychology Research and Practice, 17 (6), 565-569

Rogers, C. (1959). A theory of therapy, personality, and interpersonal relationships as developed in the client-centered framework. In J. S. Koch (Org.), Psychology: a study of a science: formulations of the person in the social context (Vol.3, pp.184-286). New York: McGraw-Hill.

Safran, J. D. (2002). Ampliando os limites da terapia cognitiva: o relacionamento terapêutico, a emoção e o processo de mudança. Porto Alegre: Artmed.

Viera, J. A. S (1994). Fundamentos del razonamiento estadistico. Porto Rico: Centro Caribeño de Estudios Postgraduados.

Yip, K. (2005) A strengths perspective in understanding and working with clients with psychosis and records of violence. Journal of Humanistic Psychology, 45 (4), 446-464.

Young, J. E., Klosko, J. S., \& Weishaar, M. E. (2003). Schema therapy. A practitioner's guide. New York: The Guilford Press.

Zillman, D. (1991). Empathy affect from bearing witness to the emotions of others. In J. Bryant \& D. Zillmann (Orgs.), Responding to the screen: reception and reaction process communication (pp.135-167). Hillsdale, NJ: Erlbaum Associates.

Recebido em: 27/7/2006

Versão final reapresentada: 15/12/2006

Aprovado em: 17/1/2007 
